They propose that inherited mutations in COQ2 cause a primary glomerular disease"COQ2 nephropathy" - characterized by renal lesions of varying severity and proliferation of dysmorphic mitochondria, but not necessarily neurological manifestations.

Patients were between 6 months and 2 years of age when studied. They had presented with isolated nephrotic syndrome, steroid-resistant nephrotic syndrome (associated with progressive encephalomyopathy in one case) or neonatal renal failure. Genetic analyses detected a homozygous c437G $>A$ mutation, two homozygous c890A $>\mathrm{G}$ mutations (harbored by a sibling pair, who had previously been reported), and a combined heterozygous mutation (c590G $>A$ and $c 683 A>G)$. Electron microscopy of renal tissue from all four patients revealed increased numbers of abnormal mitochondria in glomerular cells, particularly podocytes. Biochemical analysis of skeletal muscle and renal cortex showed decreased activity of respiratory chain complexes II and III and low levels of $\mathrm{CoQ}_{10}$. COQ2 mutations were absent from the 500 control DNA samples analyzed, and from four patients with $\mathrm{CoQ}_{10}$ deficiency but no renal involvement.

Early identification of COQ2 nephropathy could, say the authors, enable prompt initiation of ubiquinone supplementation.

Original article Diomedi-Camassei F et al. (2007)

COQ2 nephropathy: a newly described inherited mitochondriopathy with primary renal involvement. J Am Soc Nephrol 18: 2773-2780

\section{New treatment algorithms for idiopathic glomerular disease}

The calcineurin inhibitor ciclosporin is known to be an effective treatment for idiopathic glomerular disease associated with the nephrotic syndrome (INS); however, cohesive guidelines for the use of this agent in patients with INS are lacking.

An international panel of experts has evaluated the merit of clinical data on the use of ciclosporin in the three most common histological variants of INS - minimal change disease (MCD), focal segmental glomerulosclerosis (FSGS) and membranous nephropathy. The algorithms for adults and children that they have developed-which include guidance on who, when and how to treat, plus treatment goals and recommendations for follow-up - have been published in Kidney International.

Cattran et al. based their guidelines on the following findings. Ciclosporin successfully induced remission of proteinuria in approximately $80 \%$ of steroid-sensitive cases of MCD. Ciclosporin also induced remission and preserved long-term renal function in steroiddependent and steroid-resistant MCD and in steroid-resistant FSGS. The response rate in FSGS was lower than in MCD, however, and the authors recommend that ciclosporin therapy should continue for at least 12 months in patients with FSGS. Ciclosporin also lowered proteinuria in $70-80 \%$ of patients with steroid-resistant membranous nephropathy but, similarly, long-term therapy of at least 1 year was required. Although ciclosporin is generally safe, clinicians need to be vigilant to the risk of nephrotoxicity and should monitor renal function carefully during therapy.

Original article Cattran DC et al. (2007) Cyclosporin in idiopathic glomerular disease associated with the nephrotic syndrome: workshop recommendations. Kidney Int [doi:10.1038/sj.ki.5002553]

\section{Tacrolimus effective in steroid-dependent minimal change nephrotic syndrome}

A recent study found that a 24-week course of oral tacrolimus (along with tapering doses of prednisone) might be an effective alternative to ciclosporin in Chinese adults with steroiddependent minimal change disease, and might induce remission more quickly than ciclosporin.

The study enrolled 26 Chinese adults with biopsy-proven minimal change disease, steroid-dependent nephrotic syndrome, and a serum creatinine level $<133 \mu \mathrm{mol} / \mathrm{l}$ $(1.5 \mathrm{mg} / \mathrm{dl})$. Patients were self-assigned to at least 24 weeks of oral tacrolimus $(n=12$; target trough level $4-8 \mathrm{ng} / \mathrm{ml}$ ) or intravenous ciclosporin ( $n=14 ; 750 \mathrm{mg} / \mathrm{m}^{2}$ body surface area once every 4 weeks). All patients also received oral prednisone $0.5 \mathrm{mg} / \mathrm{kg} / \mathrm{day}$, tapered to cessation after complete remission (i.e. normalization of proteinuria).

One patient in each group discontinued therapy before completing 24 weeks because of severe drug-related adverse effects 